\section{Ciprofloxacin bei Gonorrhö immer seltener wirksam}

\begin{abstract}
Die Gonorrhö wird in Europa immer häufiger nachgewiesen. Aktuelle mikrobiologische Auswertungen zeigen bei den isolierten Stämmen eine deutliche Zunahme der Resistenzen gegen Ciprofloxacin. Bei Cephalosporinen der dritten Generation war bislang hingegen keine Resistenzentwicklung zu beobachten.
\end{abstract}

n den Niederlanden ist die Gonorrhö die zweithäufigste sexuell übertragene bakterielle Infektion. Im Jahr 2007 wurden dort 1.827 Infektionsfälle gemeldet. Zwei Drittel der Erkrankungen betraf homosexuelle Männer. Bei 14\% der gemeldeten Fälle bestand zugleich eine HIV-Infektion. Bei Frauen kann eine Infektion mit Gonokokken eine Urethritis, Cervicitis oder eine Entzündung des kleinen Beckens („pelvic inflammatory disease“, PID) verursachen. Als bekannte Komplikationen der durch eine Gonorrhö verursachten PID gelten die ektope Schwangerschaft, Infertilität und Aborte. Unter dem Aspekt des öffentlichen Gesundheitswesens ist eine rasche und wirksame Behandlung anzustreben, um die Dauer der Infektiosität des Erkrankten und somit die Möglichkeit der Weiterübertragung zu reduzieren.

Die WHO empfiehlt solche Antibiotika zu verwenden, die eine Wirksamkeit bei 95\% der Erkrankten sichern. Während Daten zur Inzidenz und dem Resistenzmuster bis 1999 wegen der Meldepflicht gut erfasst waren, liegen derzeit nur wenige aussagekräftige Ergebnisse vor. Beobachtungen weisen jedoch auf eine Zunahme der Resistenz gegen Fluorochinolone wie Ciprofloxacin hin. Bis in die 1990erJahre war Penicillin in der Behandlung das Mittel der ersten Wahl. Wegen zunehmender Resistenzen gegen Penicilline wurden diese seit 1997 durch eine Einmaldosis Ciprofloxacin oder Ceftriaxon ersetzt. Bedingt durch zunehmende Resistenzen gegen Ciprofloxacin wurde 2003 die Behandlungsempfehlung hin zu Cefotaxim geändert. Seit 2006 gilt nun Ceftriaxon als erstes Mittel der Wahl. Eine Auswertung der Chinolon-Resistenzen ergab eine Zunahme von 6,6\% im Jahr 2002 auf 26,4\% im Jahr 2005.

In dieser aktuellen Studie wurden nun die aktuellen Resistenzentwicklungen im
Zeitraum von 2006 bis 2008 in den Niederlanden vorgestellt. Es zeigte sich, dass $10 \%$ der isolierten Gonokokken-Stämme gegen Penicillin resistent waren, 22\% gegen Tetrazykline und $42 \%$ gegen Ciprofloxacin. Es gab keine Resistenzen gegen Cefotaxim. Bei homosexuellen Männern fanden sich höhere Resistenzen gegen Ciprofloxacin als bei heterosexuellen. Bei Frauen wiesen Prostituierte sowie Patienten, die über 35 Jahre alt waren, höhere Resistenzen gegen Ciprofloxacin auf.

Kommentar: Die Studie belegt, dass Ciprofloxacin bei der Behandlung der Gonorrhö weiterhin zunehmend an Wirksamkeit verliert, obwohl das Antibiotikum bereits seit 2003 nicht mehr zur Behandlung der Gonorrhö empfohlen wird. Die aktuellen Resistenzentwicklungen sollten - insbesondere bei einer Blindbehandlung einer Urethritis oder Cervicits - ohne Erregernachweis berücksichtigt werden. Prof. Dr. Tino F. Schwarz

Koedijk FDH et al. Increasing trend in gonococcal resistance to Ciprofloxacin in the Netherlands, 2006-2008.

Sex Transm Infect 2010; 86: 41-5

\section{Geschlechtsumwandlung: erektile Implantate im Neophallus als Herausforderung}

\section{Die Formung eines Neophallus ist der abschließende Schritt einer Geschlechtsumwandlung von der Frau zum Mann und eine große Heraus- forderung. Trotz erster enttäuschender Ergebnisse muss man aber nicht unbedingt die Flinte ins Korn werfen.}

B elgische Ärzte publizierten unlängst die Ergebnisse der bisher größten retrospektiven Studie zu hydraulischen erektilen Prothesen an insgesamt 129 Transsexuellen mit Geschlechtsumwandlung von der Frau zum Mann. Am Ende des Beobachtungszeitraumes von im Mittel 30,2 Monaten war bei gut der Hälfte der Patienten (76 von 129) die Prothese noch am Platz. Bei jedem Zweiten musste dagegen eine Revision durchgeführt werden. Die Gründe waren Infektionen,
Erosionen, eine Dysfunktion oder ein undichte Stelle. Bei einigen Patienten war es auch mit einer Revision nicht getan. Neun mussten ein weiteres Mal operiert werden, fünf zweimal, einer sogar dreimal. Daher wurden insgesamt 185 Prothesen implantiert. Die Komplikationsraten betrugen 11,9\% für Infektionen, $8,1 \%$ für Protrusionen, 9,2\% für eine undichte Stelle und 13,0\% für eine Dysfunktion. Die insgesamt höhere Komplikationsrate solcher Prothesen im Ver- gleich zu Männern mit erektiler Dysfunktion erklären die Autoren mit dem unterschiedlichen Gewebetyp des Transplantats - üblicherweise aus dem Unterarm - gegenüber dem normalen Penis.

Im Laufe der insgesamt elf Jahre, in denen sie unterschiedliche Vorgehensweisen testeten, verwendeten die Forscher verschiedene ein- und zweizylindrige Prothesen. Auch wenn die Daten dieser größten retrospektiven Untersuchung keinen wissenschaftlich fundierten Vergleich der verschiedenen Prothesenarten zulassen, so favorisieren die Autoren aufgrund ihrer Erfahrungen dennoch einzylindrige gegenüber zweizylindrigen Prothesen. wk

Hoebeke P. et al. Erectile implants in female-to-male transsexuals: our experience in 129 patients.

Eur Urol 2010; 57: 334-41 